\title{
Is immune checkpoint inhibitor-associated diabetes the same as fulminant type 1 diabetes mellitus?
}

\author{
Authors: Angelos Kyriacou, ${ }^{\mathrm{A}}$ Eka Melson, ${ }^{\mathrm{B}}$ Wentin Chen ${ }^{\mathrm{C}}$ and Punith Kempegowda ${ }^{\mathrm{D}}$
}

\begin{abstract}
Pembrolizumab is an anti-cancer drug that targets programmed cell death protein-1 (PD-1) receptors on lymphocytes resulting in their activation against tumour cells. PD-1 receptors are also interspersed in endocrine organs and pembrolizumab use has long been associated with hypophysitis and thyroiditis. Since the introduction of immune checkpoint inhibitors (ICI), several cases of fulminant type 1 diabetes mellitus (FT1DM) have been reported. However, it is unclear if FT1DM and ICI-induced diabetes are the same pathology. We review the existing literature of ICI-induced diabetes to investigate its nature and to what extent it represents type 1A diabetes and/or FT1DM (type 1B diabetes) using an example case. Our review showed that ICI-induced diabetes may be a different entity to FT1DM. Furthermore, there is limited evidence for the management of ICI-induced T1DM. Further research into its pathophysiology will improve management and possibly prevent this burdensome complication.
\end{abstract}

KEYWORDS: Diabetes, type 1, insulin-deficient, immune pathogenesis, immunopathology, ICI-induced

DOI: $10.7861 /$ clinmed.2020-0054

\section{Introduction}

Immune checkpoint inhibitors (ICI) have transformed cancer therapy by disinhibition of the immune system, with a resultant anti-neoplastic effect. However, such activation of the immune system results in complications such as hypophysitis, thyroid dysfunction, colitis and hepatitis. Type 1 diabetes mellitus (T1DM) has only recently been acknowledged as a potential side effect of these medications, and guidelines are still lagging behind this uncommon but potentially life-threatening complication.

Authors: ${ }^{\text {A }}$ consultant endocrinologist, Centre of Endocrinology, Diabetes and Metabolism, Limassol, Cyprus; ${ }^{B}$ Clinical research fellow, Institute of Metabolism and Systems Research, Birmingham, UK and University Hospitals Birmingham NHS Foundation Trust,

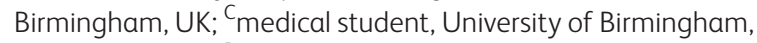
Birmingham, UK; ${ }^{\text {D }}$ Clinical research fellow, Institute of Metabolism and Systems Research, Birmingham, UK and University Hospitals Birmingham NHS Foundation Trust, Birmingham, UK
There are two main immune checkpoint systems in human, immune cells: cytotoxic T lymphocytes antigen-4 (CTLA-4) and programmed cell death protein-1 (PD-1). While CTLA-4 binds to B7 and blocks the activation of immune system at the primer stage within the lymph nodes, PD-1 binds to programmed cell death protein ligand-1 (PD-L1) to regulate the immune response at the site of action of immune system to keep the immunity in check (Fig 1). Human pancreatic islets lack CTLA-4 but instead express PD-L1 to protect them against immune cells. Pembrolizumab is a monoclonal antibody which binds to PD-1 and blocks this pathway. As a consequence, in the presence of pembrolizumab, pancreatic beta cells are susceptible to immune destruction resulting in diabetes.

Fulminant type 1 diabetes mellitus (FT1DM) is a spontaneous phenomenon characterised by markedly increased hyperglycaemia, near-normal glycated haemoglobin (HbA1c) not in line with the marked hyperglycaemia, ketoacidosis, negative autoantibodies, severe insulin deficiency and elevated levels of pancreatic enzymes. ${ }^{2}$ Since the introduction of immune checkpoint inhibitors, several cases of FT1DM have been reported. However, it is unclear if FT1DM and ICI-induced diabetes are the same pathology. In this article we review the existing literature of ICI-induced T1DM to investigate the nature of ICI-induced diabetes and to what extent it represents type $1 \mathrm{~A}$ diabetes and/or FT1DM using an example case.

\section{Case presentation}

A 68-year-old woman of South-East Asian origin presented with vomiting, dysphagia and had severe dehydration. There was no history of chest pain, palpitations, fever, cough or urinary symptoms. There was no history of similar symptoms in the past. She was diagnosed with hypertension and atrial fibrillation in the past for which she was taking losartan and metoprolol, respectively. She was also diagnosed with lung cancer (poorly differentiated non-mucinous variant) in 2016. This was initially treated with five cycles of cisplatin and pemetrexed from November 2016 until January 2017. As there was no response to the treatment, she was commenced on three weekly pembrolizumab infusions in March 2017.

In April 2017, thyrotoxicosis (free thyroxine (T4) $34 \mathrm{pmol} / \mathrm{L}$ (9-19.05 pmol/L) and thyroid stimulating hormone (TSH) 0.031 $\mathrm{mIU} / \mathrm{L}(0.35-4.94 \mathrm{mIU} / \mathrm{L}))$ was noted on routine laboratory testing. Thyroid ultrasound revealed diffuse heterogeneity and incidental sub-centimetre thyroid nodules, overall increased parenchymal 
Fig 1. Schematic representation of immune checkpoints and mechanism of action of pembrolizumab. a) Dendritic cells present antigen via histocompatibility complex (MHC) molecules which interact with $\mathrm{T}$-cell receptors (TCR). Simultaneous interaction between B7 and CD28 results in activation of T cells. b) In the absence of a programmed cell death protein-1 (PD-1) checkpoint receptor blocker, there is an interaction between PD-1 and programmed cell death protein ligand 1 (PD-L1) which prevents $T$ cell action on the cell. c) Pembrolizumab, a PD-1 receptor blocker, prevents the cancer cell-expressed PD-L1 from binding to the PD-1 receptor. This enables $T$ cells to act on the antigen presented through $\mathrm{MHC}$ and destroy the cell. Unfortunately, the beta cells in the pancreas undergo the same process of destruction as an adverse effect.

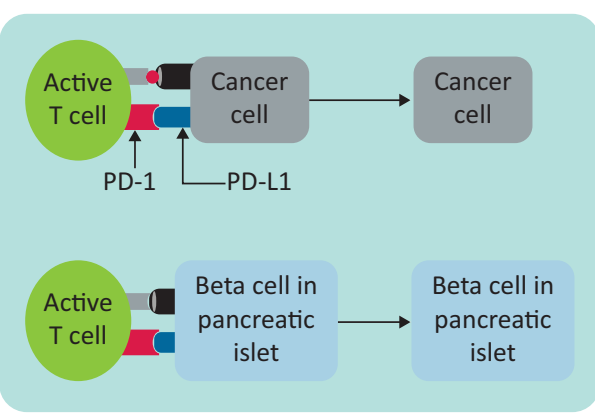

a
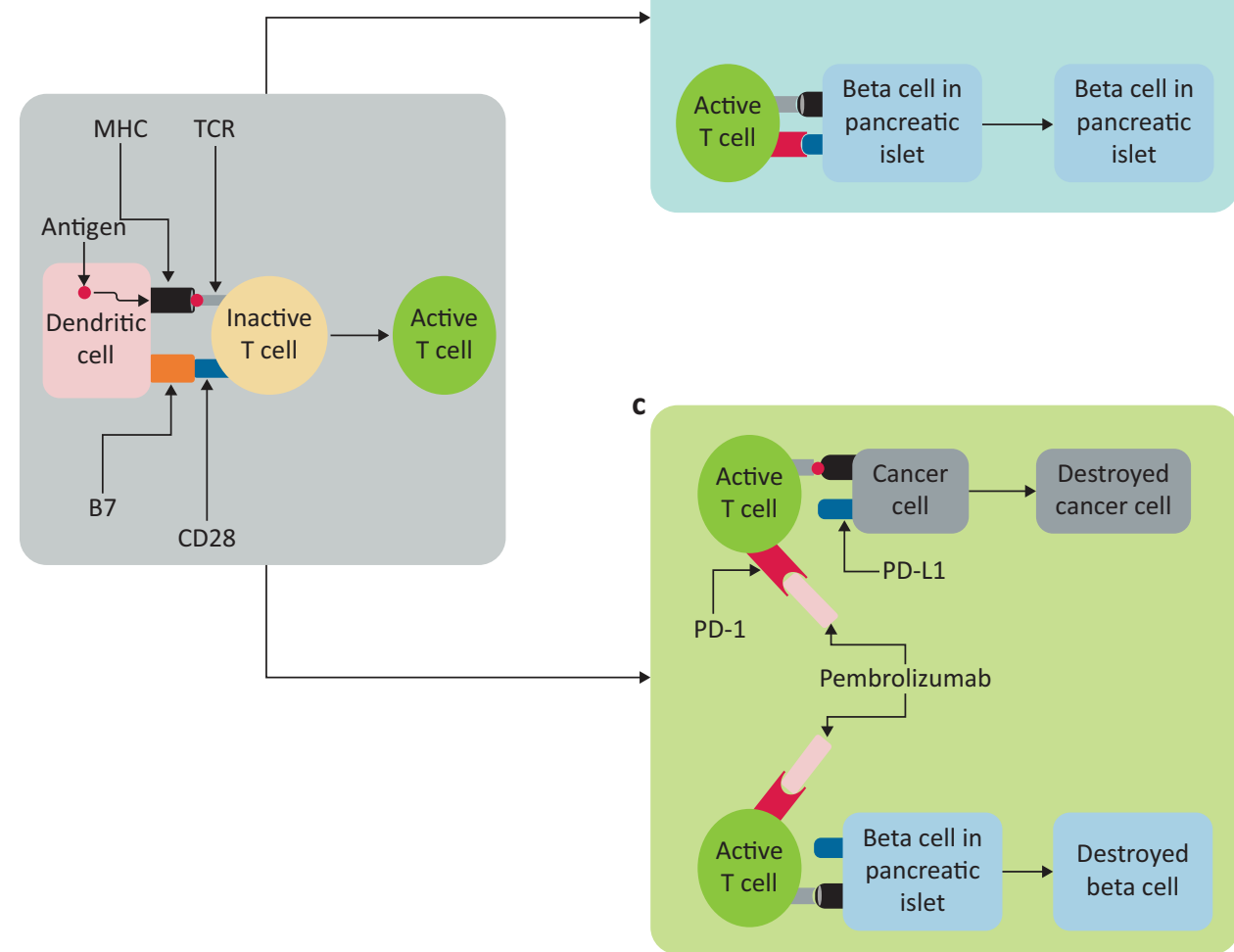

vascularity and associated reactive lymph nodes. TSH receptor antibodies were negative $(0.75 \mathrm{IU} / \mathrm{L}(<1.5 \mathrm{IU} / \mathrm{L}))$. She was clinically well and the only symptoms she offered on direct questioning were nervousness and tremor. Following evaluation, she was diagnosed with probable pembrolizumab-induced thyroiditis and managed conservatively. Her free T4 peaked at $54.3 \mathrm{pmol} / \mathrm{L}$ with free triiodothyronine (T3) $9.43 \mathrm{pmol} / \mathrm{L}$ (3.5-6.2 pmol/L) and TSH remained suppressed at $0.03 \mathrm{mIU} / \mathrm{L}$ before spontaneous improvement. However, she progressed to hypothyroidism in June 2017 (free T4 9.17 pmol/L; TSH 29.4 mIU/L). Her thyroid function has since been well controlled on levothyroxine $75 \mu \mathrm{g} /$ day.

The remainder of her pituitary function were normal except relatively low baseline morning cortisol with values between 100-150 nmol/L on different occasions with coupled adrenocorticotropic hormone (ACTH) at 6-14 pg/mL (5-35 pg/mL). Subsequent short synacthen tests did not reveal any adrenocortical insufficiency (cortisol 551.8, 874.6 and 1,109 nmol/L at baseline, 30 and 60 minutes post-ACTH injection, respectively). Her serum glucose was normal on multiple occasions between 4.4-5.5 mmol/L. Until the time of index presentation, she had received 18 months of immune checkpoint inhibitor therapy.

On examination, she was afebrile, tachycardic and hypotensive. She did not have any clinical signs of infection or systemic illness. Bedside investigations showed metabolic acidosis ( $\mathrm{pH} 7.062$ ), hyperglycaemia (serum glucose $49 \mathrm{mmol} / \mathrm{L}$ ) and ketonuria (urine ketones ++++ ). Laboratory investigations revealed acute kidney injury (urea $58.9 \mathrm{mmol} / \mathrm{L}$ (6.07-15.35 mmol/L), creatinine $231.61 \mu \mathrm{mol} / \mathrm{L}(45.08-83.98 \mu \mathrm{mol} / \mathrm{L})$, low sodium (127 mmol/L (134-144 mmol/L) and high potassium $(5.7 \mathrm{mmol} / \mathrm{L}$ (3.5-5 mmol/L). A septic screen, which included urine, blood cultures and chest X-ray, did not reveal any evidence of infection. Serum amylase and lipase were within normal limits as well (84 U/L (28-100 U/L) and $63 \mathrm{U} / \mathrm{L}$ (21-67 U/L), respectively). Following evaluation, she was diagnosed with diabetes-related ketoacidosis (DKA) and treated accordingly. She gradually improved and was discharged from hospital after 7 days on regular insulin and education regarding diabetes including dietary input.

Subsequent tests revealed supressed C-peptide at $<33.1 \mathrm{pmol} / \mathrm{L}$ (330-1,400 pmol/L) on two different occasions, indicative of a severe insulin secretion deficiency from pancreatic beta-cells. Autoantibody screen for diabetes was negative (IA-2 antibodies $4.5 \%(<6.5 \%)$, glutamic acid decarboxylase (GAD) 65 antibodies $0.01 \mathrm{IU} / \mathrm{mL}(<1 \mathrm{IU} / \mathrm{mL})$, zinc transporter 8 antibodies $2.1 \mathrm{RU} / \mathrm{mL}$ $(<15 \mathrm{RU} / \mathrm{mL}))$. Her $\mathrm{HbA} 1 \mathrm{c}$ was $53 \mathrm{mmol} / \mathrm{mol}$ (7\%). These results, alongside those from her initial presentation suggest a diagnosis of pembrolizumab-associated diabetes mellitus.

To date, she remains well on multiple daily insulin ( 8 units of insulin glulisine with meals and 18 units insulin glargine at night) albeit with an overall suboptimal glycaemic control. Her recent capillary blood glucose ranges were $4.0-16.7 \mathrm{mmol} / \mathrm{L}$ (72-300 $\mathrm{mg} / \mathrm{dL}$ ), with significant glucose fluctuations reminiscent of 
long-standing T1DM, and her $\mathrm{HbA} 1 \mathrm{c}$ was $61.7 \mathrm{mmol} / \mathrm{mol}$ (7.8\%). Pembrolizumab was recommenced following her hospital discharge but it was eventually discontinued in October 2018 due to presumed lack of efficacy (disease progression on computed cosmography (CT)) coupled with the development of drug-induced arthritis with intense joint aches limiting her mobility. She has since been on docetaxel. She received 26 cycles of pembrolizumab in total before discontinuation.

\section{Methods}

We undertook a focused, qualitative review with literature searches in PubMed, Medline and Google Scholar using a broad range of combinations of the medical subject headings (MeSH) terms: 'immune-complex inhibitor*', 'nivolumab', 'pembrolizumab', 'ipilimumab', 'programmed death*', 'programmed cell death*', 'diabetes", 'type 1 diabetes" and 'fulminant diabetes"'. Inclusion criteria were 'human' and 'abstract available' and articles retrieved from 2004 to November 2019. References of articles included were scrutinised to identify any further articles that were missed from the above database searches and personal archived references were also sought. Exclusion criterion was duplication of data. There were no other limitations regarding treatment modality, study design or study setting.

We identified all ICI-associated diabetes cases and compared them based on their diabetes-related antibody (DR-AB) status, demographics, laboratory and clinical parameters to the diagnostic criteria for FT1DM by the Japan Diabetes Society. In this diagnostic criterion, the following needed to be met to be diagnosed with FT1DM: occurrence of diabetes ketosis or ketoacidosis (approximately 7 days) after onset of hyperglycaemic symptoms; plasma glucose level $\geq 16.0 \mathrm{mmol} / \mathrm{L}$ ( $\geq 288 \mathrm{mg} / \mathrm{dL}$ ) and $\mathrm{HbA} 1 \mathrm{c}<8.7 \%$ at first visit; urinary C-peptide excretion $<10 \mu \mathrm{g} /$ day or fasting serum C-peptide level $<0.3 \mathrm{ng} / \mathrm{mL}(<0.10 \mathrm{nmol} / \mathrm{L})$ and $<0.5 \mathrm{ng} / \mathrm{mL}(<0.17 \mathrm{nmol} / \mathrm{L}$ ) after intravenous glucagon (or after meal) load at onset. ${ }^{3}$

Due to the relatively low prevalence of ICI-induced diabetes, there was heterogeneity in work-up leading to the diagnoses. Poor documentation and missing tests meant that most cases of FT1DM in the literature were labelled as non-FT1DM. Therefore, we modified the Japanese FT1DM diagnostic criteria to be more applicable to these reported cases. In our modified criteria, we removed 'the 7 days after onset of hyperglycaemic symptoms' in the first criterion. In addition, we removed the need for postglucagon stimulation C-peptide $<0.5 \mathrm{ng} / \mathrm{mL}$ as most reports did not document this information.

Descriptive statistics are provided as a median or range for continuous variables and $n(\%)$ for nominal variables. $\chi^{2}$ or Fisher's exact tests were performed as appropriate for comparison between nominal variables and Mann-Whitney $U$ test for comparison between nominal and continuous variables. All analyses were performed on SPSS v20 (IBM, New York, USA).

\section{Cases identified and descriptive statistics}

We identified 75 cases of ICI-induced diabetes. ${ }^{4-56}$ Three were excluded from analysis: one duplicate and two others due to insufficient information. ${ }^{57-59}$ Seventy-two cases of ICI-induced diabetes were included in our analysis. The median age of the patients was 63 years. There were more male participants (male:female 1.1:1). Most of the cases did not define ethnicity

of the patients $(77.8 \% ; 56 / 72)$, six patients were Japanese (8.3\%), two were non-Japanese East Asian (2.8\%), two were black American (2.8\%) and six were white (8.3\%). Most (91.7\%; $66 / 72$ ) did not have a history of diabetes. More than half of the patients $(54.2 \%$; 39/72) were started on ICI for melanoma, $29.2 \%$ $(21 / 72)$ for lung cancer, $5.6 \%$ (4/72) for renal cell carcinoma, $4.2 \%(3 / 72)$ for lymphoma and $6.9 \%$ (5/72) for other cancers. Nivolumab was the most commonly used ICI $(52.8 \% ; 38 / 72)$, followed by pembrolizumab ( $25 \%$; $18 / 72)$, ipilimumab $(4.2 \%$; $3 / 72)$ and durvalumab $(1.4 \% ; 1 / 72)$. Seven patients $(9.7 \%)$ were treated with a combination of ipilimumab plus either nivolumab or pembrolizumab, one patient (1.4\%) was in a blinded clinical trial setting (nivolumab or ipilimumab) and the ICI was not defined in one case $(1.4 \%)$.

Of the 72 cases, $43.1 \%$ (31/72) fulfilled all three diagnostic criteria for FT1DM as per the modified Japanese criteria. The breakdown of cases that fulfilled individual criteria and their combination is detailed in Table 1.

Elevated levels of pancreatic enzymes were associated with F1TDM-positive status $\left(\chi^{2} p=0.032\right)$. High levels of pancreatic enzymes were observed in $71.4 \%$ of patients diagnosed with FT1DM. There were no associations between FT1DM status and age, gender, ethnicity, personal history of diabetes, underlying malignancy, types of ICI used, number of cycles of ICI, timing of DKA/ketosis, continuation of insulin or T1D antibody status. As expected, there were positive associations between the FT1DM status and glucose $(p=0.004), \mathrm{HbA1c}(p=0.015)$ and C-peptide levels $(p<0.0001)$

Patients with negative diabetes-related autoantibodies (DRAbs) were more likely to have high pancreatic enzymes $(63.2 \%$; $12 / 19)$ compared to those with positive DR-Abs (28.6\%; 2/7). They were also more likely to fulfil the modified Japanese criteria for FT1DM $(51.3 \%$; 20/39) compared with those with positive DR-Abs (34.4\%; 11/32); however, these associations were not significant (Fisher's $p=0.19$ and $p=0.229$, respectively). There were positive associations between DR-Ab status and number of cycles of ICI $(p=0.007)$ and DKA $(p=0.016)$. No associations were found between DR-Ab status and age, gender, ethnicity, personal history of diabetes, underlying malignancy, types of ICI used,

Table 1. Proportion of cases that met individual and combination criteria for modified Japanese criteria for FT1DM.

\section{Modified Japanese criteria for FT1DM}

Occurrence of diabetes ketosis or ketoacidosis

Plasma glucose level $\geq 16.0 \mathrm{mmol} / \mathrm{L}$ ( $\geq 288 \mathrm{mg} / \mathrm{dL}$ ) and $\mathrm{HbA} 1 \mathrm{c}<8.7 \%$ at first presentation

Urinary C-peptide excretion $<10 \mu \mathrm{g} /$ day or fasting serum C-peptide level $<0.3$ $\mathrm{ng} / \mathrm{mL}(<0.10 \mathrm{nmol} / \mathrm{L})$

All the above $31(43.1)$

Cases that fulfil the criteria, $\mathrm{n}(\%)$

$56(77.8)$

$49(68.1)$

FT1DM = fulminant type 1 diabetes mellitus. 
continuation of insulin, glucose levels, HbA1c and C-peptide (preand post-glucagon stimulation test).

\section{Discussion}

Immunotherapy with checkpoint inhibitors has revolutionised cancer treatment, with six ICIs approved to treat advanced solid tumour and haematological malignancies since $2011 .{ }^{60}$ However, ICI therapy frequently induces immune-related adverse events (IRAEs) affecting multiple organ systems, most commonly endocrine. ${ }^{18,60,61}$ A study reported new-onset insulin-dependent diabetes developed after a median of four cycles or $4-5$ months since ICI initiation. Unlike other IRAEs, endocrine disruption tends to be irreversible, requiring lifelong hormone replacement and leading to hospitalisation and mortality if undetected. ${ }^{60}$

FT1DM is characterised by the absence of insulitis and diabetes-related autoantibodies including islet-cell, GAD or insulin antibodies, and elevated serum pancreatic enzyme concentrations. ${ }^{2,62}$ A meta-analysis of ICI cancer clinical trials concluded a $0.2 \%$ frequency of FT1DM, while another study reported a frequency of $1 \%{ }^{61,63}$ It should be noted that most studies determined frequency based on patients referred for concern of ICI-induced diabetes. Hence, its frequency has not been well addressed in practice. A nationwide survey reported that FT1DM accounts for $20 \%$ of T1DM cases in Japan; however, few cases have been described in Western countries. ${ }^{64,65}$

FT1DM has similarities and differences compared with spontaneous T1DM. Similarities include the frequent occurrence of DKA soon after hyperglycaemic symptoms, suggesting a faster rate of $\beta$-cell failure, and low or undetectable $C$-peptide levels which implies a lack of endogenous insulin production. ${ }^{2,33,62,63}$ FT1DM has a remarkably abrupt onset compared with autoimmune T1DM. ${ }^{63}$ FT1DM presents at an older age, with few patients presenting before 20 years old, suggesting that it is an adult-onset disease. One study reported a median age of 61 years, with adult onset observed in $>90 \%$ patients. The body mass index of patients with FT1DM is higher than the lean body habitus of patients with autoimmune T1DM and a lower weight loss is observed. ${ }^{2,63}$ Possible risk factors include specific alleles of human leukocyte antigen and combined use of checkpoint inhibitors. ${ }^{7,62}$ A study found that, in those with FT1DM, $17 \%$ and $33 \%$ had a personal or family history of autoimmunity, respectively, suggesting that ICI therapy may trigger FT1DM in patients with relevant genetic backgrounds. Another study concluded that there is no link to diabetes in firstdegree relatives, but autoimmune diseases were more frequently observed in autoimmune diabetes than in fulminant diabetes. ${ }^{64}$

In our study, most patients who developed FT1DM were being treated for melanoma. This is consistent with the most recent systematic review of ICI-induced T1DM which reported up to $53.5 \%$ of all cancers being treated by ICI. ${ }^{66}$ With the proven efficacy of ICI in the treatment of melanoma and other cancers (both as single ICI or combination), the incidence of ICIinduced FT1DM is expected to increase significantly. ${ }^{67}$ From our review, the antibody pattern coupled with pancreatic enzyme pattern observed in such patients suggests the diabetes 'type' lies closer to FT1DM (type 1B) rather than the classic type1A. At the same time, we have shown that the majority of such patients do not fulfil the classic criteria or even our modified, less confining Japanese criteria for FT1DM. Therefore, we may be dealing with a different diabetes entity altogether or, alternatively, a spectrum of diabetes encompassing both type $1 \mathrm{~A}$ and $1 \mathrm{~B}$, that may be better named as ICI-induced diabetes or insulin-dependent diabetes secondary to ICI. T1DM is usually associated with at least one positive DR-Ab by the time of diabetes presentation. ${ }^{62}$ This misclassification may have led to a heterogeneity in the work-up leading to diagnosis and therefore classifying this condition could be the first step in improving our understanding of the pathophysiology of this condition. A better understanding of the pathophysiology which, in turn, could aid in better screening and monitoring for the development and treatment of ICI-induced diabetes and perhaps even the prevention or stratification of patients regarding their risk this complication before commencing treatment with ICI. A retrospective study by Kotwal et al looking at 1,444 patients treated with ICI identified several potential predictive factors that could influence the occurrence and severity of ICI-induced diabetes. Personal and family history of autoimmunity were associated with this adverse event with $17 \%$ and $33 \%$ of the ICI-induced diabetes cases having a positive personal and family history of autoimmune conditions, respectively. Almost two-thirds of the 1,444 patients had another IRAE suggesting that presence of one IRAE may predict the development of another. ${ }^{63}$ Patients on a combination of immunotherapies and/ or pre-treatment with other immunotherapies before starting ICI also appeared to be at an increased risk of ICI-induced diabetes. ${ }^{61,68}$ Pre-existing type 2 diabetes did not appear to predict occurrence. ${ }^{52}$ It is however important to note that these studies are mostly cross-sectional and retrospective in nature. Prospective studies are needed before any conclusions can be drawn regarding the risk factors of developing ICIinduced diabetes. Noguira et al suggested strategies in the assessment, management and monitoring of immunotherapyinduced endocrinopathies. Due to the rapid onset of ICIinduced diabetes, all acutely unwell patients on ICI should have their blood glucose checked and/or a full work-up for DKA, if necessary. Patients should be treated with insulin and hospitals should ensure that there must be a system in place to ensure safe administration of insulin. They should also be referred to the diabetes team for further education and management. ${ }^{60}$ Furthermore, there is limited evidence for the management of ICI-induced T1DM and most of the cases have been managed acutely when presenting with DKA as per standard DKA protocol and with T1DM in the intermediate- and long-term. To the best of our knowledge, there are no studies comparing the dose requirements of insulin in this cohort. However, from our experience and review of literature, the major difference between ICI diabetes and classic T1DM is the lack of a 'honeymoon period', a brief phase sometimes seen in classic T1DM during which they get better with a minimal need for insulin therapy. ${ }^{69}$ There is also no strong evidence whether to stop or change the ICI with the American Society of Clinical Oncology guideline suggesting to restart the ICI after the resolution of hyperglycaemia. ${ }^{70}$ However, this has been challenged by some reports that withdrawal of the ICI would preserve pancreatic insulin capacity. ${ }^{71}$ With the lack of evidence, more observational studies and/or clinical trials are needed to improve the overall approach to this condition.

We further compared our findings with two large case series in this field (Table 2) ${ }^{43,62}$ In all series, the median age at diagnosis was much higher than classic T1DM presentation with no apparent 
Table 2. Comparison of our results with the case series from Clotman et al and Stamatouli et al. ${ }^{43,62}$

\begin{tabular}{|c|c|c|c|}
\hline Parameter & $\begin{array}{l}\text { Current study } \\
(n=72)\end{array}$ & $\begin{array}{l}\text { Clotman study } \\
(n=41)\end{array}$ & $\begin{array}{l}\text { Stamatouli study } \\
(n=27)\end{array}$ \\
\hline Median age, years (IQR) & $63(22-83)$ & $63(28-83)$ & $66(49-87)$ \\
\hline Gender, \% (male:female) & $52.8: 47.2$ & $51.2: 48.8$ & 62.9:37.1 \\
\hline Index presentation with DKA or ketosis, $\%$ & 77.8 & 71.4 & 59.0 \\
\hline Diabetes-related autoantibodies, \% & 44.4 & 56.0 & 40.0 \\
\hline GAD positivity, \% & 39.4 & 56.0 & NCD \\
\hline HbA1c, mmol/mol (IQR) & $53(39.9-101.1)$ & $53(46.4-93.4)$ & $63.4(42.1-90.2)$ \\
\hline Proportion of cases with reduced C-peptide $<1.1 \mathrm{ng} / \mathrm{mL}, \%$ & 62.5 & 93.0 & 85.0 \\
\hline HLA genotypes, \% & NCD & NCD & $\begin{aligned}> & \text { HLA-DR4: } 76 \\
> & \text { HLA-DR3: } 34.1 \\
> & \text { HLA-DQ8 } \\
& \text { (DQB1*0302): } 38.0\end{aligned}$ \\
\hline $\begin{array}{l}\text { Proportion of patients in whom ICI therapy continued after } \\
\text { diagnosing diabetes, \% }\end{array}$ & 41.7 & NCD & 37.0 \\
\hline $\begin{array}{l}\text { Proportion of patients treated with insulin for ICI-induced } \\
\text { diabetes, \% }\end{array}$ & 98.6 & NCD & NCD \\
\hline
\end{tabular}

$\mathrm{DKA}=$ diabetes-related ketoacidosis; $\mathrm{GAD}=$ glutamic acid decarboxylase; HbA1c = glycated haemoglobin; HLA = human leukocyte antigen; ICI = immune checkpoint inhibitor; IQR = interquartile range; NCD = not clearly documented.

gender preponderance. There was a much higher frequency of DKA or ketosis at diagnosis than standard T1DM cases. A majority of the patients were diabetes-related Ab negative, unlike T1DM. The median $\mathrm{HbA} 1 \mathrm{C}$ was low suggesting abrupt onset of diabetes. Remarkably, C-peptide levels were undetectable in a majority of cases in the Clotman and Stamatouli series. However, once we applied stricter criteria for FT1DM, less than two-thirds had significantly reduced C-peptide. In the case series by Stamatouli et al, frequency of HLA-DR3 was higher than the general population for spontaneous T1DM. HLA-DR3 and HLA-DQ8 $\left(\mathrm{DQB1}{ }^{*} 0302\right)$ was also higher than general population but similar to T1DM prevalence. In the Clotman et al series, 67\% (14/21) had HLA haplotype which is high risk for T1DM. However, the recording of these haplotypes was not consistent between studies. It is unclear what proportion of patients needed discontinuation of ICI therapy after being diagnosed with diabetes as an adverse effect. Further well-designed studies are needed to clarify this. Although it was not clearly documented what proportion of patients needed long term insulin, our series suggest there was no "honeymoon period' in ICI-associated diabetes cases, which differentiated them from classic T1DM cases.

Our review and other studies, have consistently observed that the rate of pancreatitis in those with DKA (depicted by a raised pancreatic enzymes) is higher than the general population of ICI-treated patients; however, this was not the case with our patient. ${ }^{72,73}$ This leads to the speculation that blockade of cellular response to inflammatory mediators, possibly due to pancreatitis and/or other inflammatory processes, may be a potential driver in the disease development. Stamatouli et al also showed in their review that HLA-DR4 seems to be over-represented in patients with ICI-induced diabetes. Other high-risk alleles for spontaneous T1DM, including HLA-DR3, DQ2 and DQ8, were not overrepresented. ${ }^{62}$ Furthermore, $70 \%$ of the cases reviewed had other IRAEs and $44 \%$ had endocrine-related IRAEs prior or concurrent to the development of ICI-induced diabetes (especially primary thyroid dysfunction) which was also true of our patient. These observations may point towards potential pre-treatment screening that might be needed to identify those who are at a higher risk of developing ICI-induced diabetes following treatment with ICI. However, more studies are needed to prove these hypotheses.

\section{Conclusion}

Our review shows that ICI-induced diabetes is a different entity to FT1DM, at least for the majority of patients with this condition. We have also demonstrated that the patient subgroup with DR-Ab negativity behaves differently with a significant, albeit modest, increase in the risk of DKA and a presentation with ICI-induced diabetes after more cycles of ICI. Further research into the pathophysiology of this condition will aid in the better management and possibly the prevention of this burdensome complication.

\section{References}

1 Shieh S-J, Chou F-C, Yu P-N et al. Transgenic expression of singlechain anti-CTLA-4 Fv on $\beta$ cells protects nonobese diabetic mice from autoimmune diabetes. J Immunol 2009;183:2277-85.

2 Imagawa A, Hanafusa T, Miyagawa J, Matsuzawa Y. A novel subtype of type 1 diabetes mellitus characterized by a rapid onset and an absence of diabetes-related antibodies. N Engl J Med 2000;342:301-7.

3 Imagawa A, Hanafusa T, Awata T et al. Report of the Committee of the Japan Diabetes Society on the research of fulminant and acute-onset type 1 diabetes mellitus: new diagnostic criteria of fulminant type 1 diabetes mellitus (2012). J Diabetes Investig 2012;3:536-9.

4 Okamoto M, Okamoto M, Gotoh K et al. Fulminant type 1 diabetes mellitus with anti-programmed cell death-1 therapy. J Diabetes Investig 2016;7:915-8. 
5 Gaudy C, Clévy C, Monestier S et al. Anti-PD1 pembrolizumab can induce exceptional fulminant type 1 diabetes. Diabetes Care 2015;38:e182-3.

6 Takahashi A, Tsutsumida A, Namikawa K, Yamazaki N. Fulminant type 1 diabetes associated with nivolumab in a patient with metastatic melanoma. Melanoma Res. 2018;28:159-60.

7 Hughes ], Vudattu N, Sznol M et al. Precipitation of autoimmune diabetes with anti-PD-1 immunotherapy. Diabetes Care 2015:38:e55-7.

8 Munakata W, Ohashi K, Yamauchi N, Tobinai K. Fulminant type I diabetes mellitus associated with nivolumab in a patient with relapsed classical Hodgkin lymphoma. Int $\mathrm{J}$ Hematol 2017;105:383-6.

9 Kong SH, Lee SY, Yang YS, Kim TM, Kwak SH. Anti-programmed cell death 1 therapy triggering diabetic ketoacidosis and fulminant type 1 diabetes. Acta Diabetol 2016;53:853-6.

10 Lowe JR, Perry DJ, Salama AKS et al. Genetic risk analysis of a patient with fulminant autoimmune type 1 diabetes mellitus secondary to combination ipilimumab and nivolumab immunotherapy. J Immunother Cancer 2016;4:89.

11 Teramoto Y, Nakamura Y, Asami Y et al. Case of type 1 diabetes associated with less-dose nivolumab therapy in a melanoma patient. J Dermatol 2017:44:605-6.

12 Miyoshi Y, Ogawa O, Oyama Y. Nivolumab, an anti-programmed cell death-1 antibody, induces fulminant type 1 diabetes. Tohoku J Exp Med 2016;239:155-8

13 Sakurai K, Niitsuma S, Sato R, Takahashi K, Arihara Z. Painless thyroiditis and fulminant type 1 diabetes mellitus in a patient treated with an immune checkpoint inhibitor, nivolumab. Tohoku J Exp Med 2018;244:33-40.

14 Shiba M, Inaba H, Ariyasu $\mathrm{H}$ et al. Fulminant type 1 diabetes mellitus accompanied by positive conversion of anti-insulin antibody after the administration of anti-CTLA-4 antibody following the discontinuation of anti-PD-1 antibody. Intern Med 2018;57:2029-34.

15 Tsiogka A, Jansky GL, Bauer JW, Koelblinger P. Fulminant type 1 diabetes after adjuvant ipilimumab therapy in cutaneous mela noma. Melanoma Res 2017:27:524-5

16 Mizab Mellah C, Sánchez Pérez M, Santos Rey MD, Hernández García M. Diabetes fulminante secundaria a tratamiento con pembrolizumab. Endocrinol Diabetes y Nutr 2017;64:272-3.

17 Araújo M, Ligeiro D, Costa $L$ et al. A case of fulminant Type 1 diabetes following anti-PD1 immunotherapy in a genetically susceptible patient. Immunotherapy 2017:9:531-5.

18 Ishikawa K, Shono-Saito T, Yamate T et al. A case of fulminant type 1 diabetes mellitus, with a precipitous decrease in pancreatic volume, induced by nivolumab for malignant melanoma: analysis of HLA and CTLA-4 polymorphisms. Eur ] Dermatology 2017;27:184-5.

19 Martin-Liberal J, Furness AJ, Joshi K et al. Anti-programmed cell death-1 therapy and insulin-dependent diabetes: a case report. Cancer Immunol Immunother 2015;64:765-7.

20 Chae YK, Chiec L, Mohindra N et al. A case of pembrolizumabinduced type-1 diabetes mellitus and discussion of immune checkpoint inhibitor-induced type 1 diabetes. Cancer Immunol Immunother 2017;66:25-32.

21 Hofmann L, Forschner A, Loquai $C$ et al. Cutaneous, gastrointestinal, hepatic, endocrine, and renal side-effects of anti-PD-1 therapy. Eur J Cancer 2016;60:190-209.

22 Hansen E, Sahasrabudhe D, Sievert L. A case report of insulindependent diabetes as immune-related toxicity of pembrolizumab: presentation, management and outcome. Cancer Immunol Immunother 2016:65:765-7.

23 Li L, Masood A, Bari S, Yavuz S, Grosbach AB. Autoimmune diabetes and thyroiditis complicating treatment with nivolumab. Case Rep Oncol 2017:10:230-4.
24 Mellati M, Eaton KD, Brooks-Worrell BM et al. Anti-PD-1 and Anti-PDL-1 monoclonal antibodies causing type 1 diabetes. Diabetes Care 2015;38:e137-8.

25 Tzoulis P, Corbett RW, Ponnampalam S et al. Nivolumab-induced fulminant diabetic ketoacidosis followed by thyroiditis. Endocrinol Diabetes Metab Case Rep 2018:2018:18-0111.

26 Teló GH, Carvalhal GF, Cauduro CGS et al. Fulminant type 1 diabetes caused by dual immune checkpoint blockade in metastatic renal cell carcinoma. Ann Oncol 2017;28:191-2.

27 Abdul Aziz MHF, Fernando IP, Lenkanpally A, Fernando DJS Diabetic ketoacidosis after treatment with pembrolizumab. J Clin Transl Endocrinol Case Rep 2017:5:4-5.

28 Fukui A, Sugiyama K, Yamada T et al. A case of nivolumabinduced fulminant type 1 diabetes with steroids and glucagon-like peptide 1 administration during the early onset. J Clin Case Rep 2016:6:883.

29 Frunza-Stefan S, Whitlatch $\mathrm{H}$. A case of nivolumab induced fulminant type 1 diabetes. Endocr Abstr. 2018;56:126.

30 Godwin JL, Jaggi S, Sirisena I et al. Nivolumab-induced autoimmune diabetes mellitus presenting as diabetic ketoacidosis in a patient with metastatic lung cancer. J Immunother Cancer 2017:5:40.

31 Zaied AA, Akturk HK, Joseph RW, Lee AS. New-onset insulindependent diabetes due to nivolumab. Endocrinol Diabetes Metab Case Rep. 2018;2018:17-0174.

32 Hickmott L, De La Peña H, Turner H et al. Anti-PD-L1 atezolizumabinduced autoimmune diabetes: a case report and review of the literature. Target Oncol 2017;12:235-41.

33 Gauci ML, Laly P, Vidal-Trecan T et al. Autoimmune diabetes induced by PD-1 inhibitor-retrospective analysis and pathogenesis: a case report and literature review. Cancer Immunol Immunother 2017:66:1399-410.

34 Thoreau B, Gouaillier-Vulcain F, Machet L et al. Acute lower limb ischaemia and diabetes in a patient treated with anti-PD1 monoclonal antibody for metastatic melanoma. Acta Derm Venereol 2017;97:408-9.

35 Usui $\mathrm{Y}$, Udagawa H, Matsumoto $\mathrm{S}$ et al. Association of serum anti-GAD antibody and HLA haplotypes with type 1 diabetes mellitus triggered by nivolumab in patients with non-small cell lung cancer. J Thorac Oncol 2017;12:e41-3.

36 Leonardi GC, Oxnard GR, Haas A et al. Diabetic ketoacidosis as an immune-related adverse event from pembrolizumab in non-small cell lung cancer. J Immunother 2017:40:249-51.

37 Scott ES, Long GV, Guminski A et al. The spectrum, incidence, kinetics and management of endocrinopathies with immune checkpoint inhibitors for metastatic melanoma. Eur ] Endocrinol 2018;178:173-80.

38 Kapke J, Shaheen Z, Kilari D, Knudson P, Wong S. Immune checkpoint inhibitor-associated type 1 diabetes mellitus: case series, review of the literature, and optimal management. Case Rep Oncol 2017;10:897-909.

39 Shah M, Maxfield L, Feroz R, Donohue K. Rapid development of type 1 diabetes mellitus after initiation of anti-PD-1 therapy. Int J Cancer Clin Res 2016:3:066.

40 Hao JB, Renno A, Imam S et al. Development of type 1 diabetes after cancer immunotherapy. AACE Clin Case Reports 2017;3: e242-5.

41 Alhusseini M, Samantray J. Autoimmune diabetes superimposed on type 2 diabetes in a patient initiated on immunotherapy for lung cancer. Diabetes Metab 2017:43:86-8.

42 Asif Humayun M, Poole R. A case of multiple immune toxicities from ipilimumab and pembrolizumab treatment. Hormones 2016;15:303-6.

43 Clotman K, Janssens K, Specenier P, Weets I, De Block CEM. Programmed cell death-1 inhibitor-induced type 1 diabetes mellitus. J Clin Endocrinol Metab 2018;103:3144-54. 
44 Aleksova ], Lau PKH, Soldatos G, McArthur G. Glucocorticoids did not reverse type 1 diabetes mellitus secondary to pembrolizumab in a patient with metastatic melanoma. BMJ Case Rep 2016;2016:bcr2016217454

45 Alzenaidi AA, Dendy J, Rejjal L. Autoimmune diabetes presented with diabetic ketoacidosis induced by immunotherapy in an adult with melanoma. J La State Med Soc 2017;169:49.

46 Marchand L, Paulus V, Fabien N et al. Nivolumab-induced acute diabetes mellitus and hypophysitis in a patient with advanced pulmonary pleomorphic carcinoma with a prolonged tumor response. J Thorac Oncol. 2017;12:e182-4.

47 Changizzadeh PN, Mukkamalla SKR, Armenio VA. Combined checkpoint inhibitor therapy causing diabetic ketoacidosis in metastatic melanoma. J Immunother Cancer 2017:5:97.

48 Villarreal J, Townes D, Vrablik M, Ro K. A case of drug-induced severe endocrinopathies what providers in the emergency department need to know. Adv Emerg Nurs J 2018:40:16-20.

49 Kumagai R, Muramatsu A, Nakajima R et al. Acute-onset type 1 diabetes mellitus caused by nivolumab in a patient with advanced pulmonary adenocarcinoma. J Diabetes Investig 2017;8:798-9.

50 Chang E, Rivero G, Patel NR et al. HIV-related refractory hodgkin Iymphoma: a case report of complete response to nivolumab. Clin Lymphoma Myeloma Leuk 2018;18:e143-6.

51 Smith-Cohn MA, Gill D, Voorhies BN, Agarwal N, Garrido-Laguna I. Case report: pembrolizumab-induced Type 1 diabetes in a patient with metastatic cholangiocarcinoma. Immunotherapy 2017:9:797-804

52 Magis Q, Gaudy-Marqueste C, Basire A et al. Diabetes and blood glucose disorders under Anti-PD1. J Immunother 2018;41: 232-40.

53 Matsumura K, Nagasawa K, Oshima Y et al. Aggravation of diabetes, and incompletely deficient insulin secretion in a case with type 1 diabetes-resistant human leukocyte antigen DRB1*15:02 treated with nivolumab. J Diabetes Investig 2018;9:438-41.

54 Tassone F, Colantonio I, Gamarra E et al. Nivolumab-induced fulminant type 1 diabetes (T1D): the first Italian case report with long follow-up and flash glucose monitoring. Acta Diabetol 2019;56:489-90.

55 Stevens E, Bhat S. New onset type 1 diabetes and diabetic ketoacidosis induced by nivolumab therapy. Proc UCLA Healthc 2017;21.

56 Marchand L, Thivolet A, Dalle S et al. Diabetes mellitus induced by PD-1 and PD-L1 inhibitors: description of pancreatic endocrine and exocrine phenotype. Acta Diabetol 2019;56:441-8.

57 Marchand L, Thivolet A, Saintigny P et al. Anti-programmed death 1 (PD-1) antibodies and the pancreas: a diabetic storm ahead? Diabetes Care 2018:41:638-9.

58 Capitao R, Bello C, Fonseca R, Saraiva C. New onset diabetes after nivolumab treatment. BMJ Case Rep 2018;2018:bcr-2017220999.

59 Brahmer JR, Tykodi SS, Chow LQM et al. Safety and activity of anti-PD-L1 antibody in patients with advanced cancer. N Engl J Med 2012;366:2455-65.
60 Nogueira E, Newsom-Davis T, Morganstein DL. Immunotherapyinduced endocrinopathies: assessment, management and monitoring. Ther Adv Endocrinol Metab 2019;10:2042018819896182.

61 Barroso-Sousa R, Barry WT, Garrido-Castro AC et al. Incidence of endocrine dysfunction following the use of different immune checkpoint inhibitor regimens a systematic review and metaanalysis. JAMA Oncology 2018;4:173-82.

62 Stamatouli AM, Quandt Z, Perdigoto AL et al. Collateral damage: insulin-dependent diabetes induced with checkpoint inhibitors. Diabetes 2018;67:1471-80.

63 Kotwal A, Haddox C, Block M, Kudva YC. Immune checkpoint inhibitors: An emerging cause of insulin-dependent diabetes. BMJ Open Diabetes Res Care 2019;7:e000591.

64 Imagawa $\mathrm{A}$, Hanafusa $\mathrm{T}$, Uchigata $\mathrm{Y}$ et al. Fulminant type 1 diabetes: A nationwide survey in Japan. Diabetes Care 2003:26:2345-52.

65 Pozzilli P, Visalli N, Leslie D. No evidence of rapid onset (Japanese) type I diabetes in Caucasian patients. Diabetologia 2000;43:1332

66 Akturk HK, Kahramangil D, Sarwal A et al. Immune checkpoint inhibitor-induced type 1 diabetes: a systematic review and metaanalysis. Diabet Med 2019;36:1075-81.

67 Wu Y, Shi H, Jiang M et al. The clinical value of combination of immune checkpoint inhibitors in cancer patients: A meta-analysis of efficacy and safety. Int J Cancer 2017;141:2562-70.

68 Byun DJ, Wolchok JD, Rosenberg LM, Girotra M. Cancer immunotherapy-immune checkpoint blockade and associated endocrinopathies. Nat Rev Endocrinol 2017;13:195-207.

69 Nwosu BU. Partial Clinical Remission of type 1 diabetes mellitus in children: clinical applications and challenges with its definitions. Eur Med J Diabetes 2019;4:89-98.

70 Brahmer JR, Lacchetti C, Schneider B] et al. Management of immune-related adverse events in patients treated with immune checkpoint inhibitor therapy: American society of clinical oncology clinical practice guideline. J Clin Oncol 2018;36:1714-68.

71 Sakai G, Saito D, Nakajima R et al. Intrinsic insulin secretion capacity might be preserved by discontinuing anti-programmed cell death protein 1 antibody treatment in "anti-programmed cell death protein 1 antibody-induced' fulminant type 1 diabetes. J Diabetes Investig 2018;9:448-9.

72 Nair S, Yadav D, Pitchumoni CS. Association of diabetic ketoacidosis and acute pancreatitis: observations in 100 consecutive episodes of DKA. Am J Gastroenterol 2000;95:2795-800.

73 Friedman CF, Clark V, Raikhel AV et al. Thinking critically about classifying adverse events: incidence of pancreatitis in patients treated with nivolumab + ipilimumab. J Nat/ Cancer Inst 2017;109:djw260.

Address for correspondence: Dr Punith Kempegowda, Institute of Metabolism and Systems Research, University of Birmingham, IBR Level 2, College of Medical and Dental Sciences, Edgbaston, Birmingham B15 2TT, UK. Email: p.kempegowda@bham.ac.uk 